INGRID FONSÊCA DAMASCENO BEZERRA ${ }^{1}$

Vanessa Patrícia SoARes de Sousa ${ }^{1}$

LeNIZE COSTA dOS SANTOS ${ }^{2}$

Elizabel de Souza Ramalho Viana ${ }^{2}$

\section{Artigo Original}

Palavras-chave

Gravidez

Gestantes

Comportamento sexual Qualidade de vida

Keywords

Pregnancy

Pregnant women

Sexual behavio

Quality of life

\title{
Comparação da qualidade de vida em gestantes com disfunção sexual
}

\section{Comparison of quality of life in women with sexual dysfunction}

\section{Resumo}

OBJETIVO: Investigar a relação entre função sexual e qualidade de vida em mulheres grávidas, residentes em duas cidades do Nordeste. MÉTODOS: A amostra constituiu-se de 207 gestantes. A coleta de dados foi feita por meio da aplicação de questionário contendo questões sobre dados sociodemográficos, ginecológicos e obstétricos, conhecimento corporal e sexual. A qualidade de vida foi avaliada através da aplicação do Índice de Qualidade de Vida Ferrans \& Powers (IQV Ferrans e Power). A função sexual foi avaliada por meio do Índice de Função Sexual Feminina (IFSF). Foram realizados os testes estatísticos de Shapiro-Wilk, Mann-Whitney e Wilcoxon para análise dos dados coletados. RESULTADOS: As gestantes avaliadas apresentaram idade mediana de 30 anos (quartil 26-33 anos) e estavam aproximadamente na $26^{\circ}$ semana gestacional. Observou-se diminuição significativa da frequência mensal do relacionamento sexual do casal, que passou de uma mediana de 12 para 4 vezes por mês $(Z=-10,56 ; p<0,001)$. A disfunção sexual se mostrou presente em 35,7\% das gestantes avaliadas, e a qualidade de vida dessas foi inferior quando comparada àquelas com função sexual sem alteração $(Z=-2,87 ; p=0,004)$. CONCLUSÃO: Os resultados do presente estudo mostram que a disfunção sexual afetou negativamente a qualidade de vida de mulheres grávidas, devendo ser um aspecto relevante para ser avaliado durante as consultas de pré-natal.

\section{Abstract}

PURPOSE: To investigate the relationship between sexual function and quality of life in pregnant women living in two cities of Northeastern Brazil. METHODS: The sample consisted of 207 pregnant women. The data were collected through a questionnaire containing questions about socio-demographic, gynecological and obstetrical data, body and sexual knowledge. Quality of life was assessed by applying the Ferrans \& Powers Quality of Life Index (QLI Ferrans and Power). Sexual function was assessed using the Female Sexual Function Index (IFSF). Data were statistically analyzed using the Shapiro-Wilk, Mann-Whitney and Wilcoxon tests. RESULTS: The pregnant women studied had a median age of 30 years (quartile 26-33 years) and were approximately at the $26^{\text {th }}$ gestational week. A significant decrease in the monthly frequency of sexual relations of the couple was observed, with a median of 12 to 4 times per month $(Z=-10.56 ; p<0.001)$. Sexual dysfunction was detected in $35.7 \%$ of the pregnant women studied, whose quality of life was lower when compared to women with unchanged sexual function ( $Z=-2.9 ; p=0.004)$. CONCLUSION: The results of this study show that sexual dysfunction negatively affected the quality of life of pregnant women, and this should be an important aspect for review during prenatal consultations.

Correspondêncio

Ingrid Fonsêca Damasceno Bezerra Departamento de Fisioterapia, Universidade Federal do Rio Grande do Norte Avenida Senador Salgado Filho, 3000 - Caixa Postal 1524 CEP: $59072-970$ Natal (RN), Brasil

Recebido $22 / 12 / 2014$

Aceito com modificacộes $11 / 05 / 2015$
Departamento de Fisioterapia da Universidade Federal do Rio Grande do Norte - UFRN - Natal (RN), Brasil.

Programa de Pós-graduação em Fisioterapia, Universidade Federal do Rio Grande do Norte - UFRN - Natal (RN), Brasil. ${ }^{2}$ Curso de Fisioterapia, Universidade Federal do Rio Grande do Norte - UFRN - Natal (RN), Brasil.

Conflito de interesses: não há. 


\section{Introdução}

Durante a gravidez, as mulheres podem ter a função sexual e a qualidade de vida $(\mathrm{QV})$ influenciadas pela forma como vivenciam o período gravídico. Em geral, esse público apresenta uma diminuição no desejo, excitação, orgasmo e lubrificação, o que pode levar ao aparecimento de dor e provocar uma insatisfação sexual. Essas alterações são normalmente identificadas em gestantes no início do processo gestacional, nas de alto risco e naquelas com idade gestacional avançada ${ }^{1,2}$.

A sexualidade humana interfere diretamente na QV do indivíduo, o que tem chamado a atenção da ciência para realização de mais estudos sobre esse tema. A sexualidade feminina sofre influência ao longo da vida por incontáveis fatores biológicos, fatos da vida ou relacionados à reprodução (gravidez, menopausa), saúde (câncer, doença crônica e medicação), relação (parceiro, intimidade) e fatores socioculturais (educação, tabus) ${ }^{3-7}$.

As disfunções sexuais ocorrem em ambos os sexos e são definidas pela falta, desconforto e/ou dor no decorrer da resposta sexual, interferindo de forma negativa em alguma fase desse evento (desejo, excitação e/ou orgasmo), podendo causar bloqueios e traumas ${ }^{7-12}$.

A disfunção sexual feminina é contextualizada como qualquer problema que interfira na resposta sexual, provocando irregularidades do funcionamento sexual normal da mulher. Isso pode causar desconforto e afetar a sua $\mathrm{QV}^{7,9,13,14}$. Durante o período gestacional, essa disfunção pode ocorrer por questões como presença de desconforto físico, receio de machucar o bebê, diminuição da libido e dor durante $o$ ato sexual ${ }^{8,15-18}$.

A relação entre a gestação e disfunção sexual tem despertado muito interesse na literatura, porém, não há dados suficientes considerando a prevalência da disfunção sexual durante a gestação. A maior dificuldade encontrada para abordar essa temática ainda é o desconforto que cerca as mulheres grávidas e os profissionais que as acompanham durante essa fase $\mathrm{e}^{5,16,19}$.

Este estudo busca verificar a comparação da QV entre gestantes com e sem disfunção sexual, uma vez que existe escassez de estudos sobre o tema e, também, pela necessidade de maior conhecimento que embase as práticas clínicas com as mulheres grávidas.

\section{Métodos}

Foi desenvolvido um estudo observacional, analítico e transversal, cuja população foi composta por mulheres grávidas entre o segundo e terceiro trimestre de gestação, no período de abril de 2013 a julho de 2014 . A amostra foi composta por gestantes que frequentavam os serviços de pré-natal do Sistema Único de Saúde (SUS) da Maternidade
Divino Amor em Parnamirim, no Rio Grande do Norte, e de mulheres que participaram do Curso para Gestantes do Departamentos de Fisioterapia da Universidade Federal do Rio Grande do Norte (UFRN), Natal, também no Rio Grande do Norte, municípios localizados na região Nordeste brasileira.

A amostra por conveniência constituiu-se de um total de 207 gestantes, distribuídas em 95 mulheres da maternidade e 112 gestantes que foram acompanhadas pelo curso de gestantes. O estudo foi aprovado pelo Comitê de Ética em Pesquisa da Universidade Federal do Rio Grande do Norte (CEP-UFRN), de acordo com a Resolução no 466/12, sob o parecer número 719.939.

Para participar do estudo, as voluntárias teriam que ter idade entre 18 e 45 anos, com exames que confirmassem a gravidez, que fossem atendidas na Maternidade Divino Amor (Parnamirim) ou que estivessem participando do curso para gestantes do departamento de Fisioterapia da UFRN (Natal), com gestação única e parceiro nos últimos seis meses, alfabetizada e com Termo de Consentimento Livre e Esclarecido assinado. As mulheres que se recusaram a responder todos os questionários de avaliação e que não apresentavam vida sexual ativa no último mês foram excluídas do estudo.

Para a realização das coletas de dados dessas mulheres foi aplicada uma ficha contento questões através das quais eram obtidas as características sociodemográficas (idade, estado civil, escolaridade e renda familiar) e dados gestacionais (planejamento da gravidez, idade gestacional, acompanhamento obstétrico). Em seguida, foram questionadas acerca dos domínios sexuais (desejo, excitação, orgasmo, dor e satisfação), antes e durante a gestação. Adicionalmente, foram indagadas sobre conhecimento corporal e educação sexual na gestação.

Com o intuito de avaliar a função sexual das voluntárias, foi aplicado o Índice de Função Sexual Feminina (IFSF), traduzido para a língua portuguesa, adaptado culturalmente para a realidade brasileira e validado para avaliar gestantes ${ }^{20}$. O IFSF é um questionário conciso, autoaplicável, e admite transformar respostas subjetivas em informações objetivas, quantificáveis e analisáveis, no que se refere à função sexual feminina. Esse protocolo se propõe a avaliar a resposta sexual feminina em seis domínios: desejo sexual, excitação sexual, lubrificação vaginal, orgasmo, satisfação sexual e dor. Esse questionário apresenta 19 questões, que avaliam a função sexual, nas últimas 4 semanas. Em cada quesito, existe um padrão de resposta, sendo que as opções recebem pontuação de 0 a 5 , de forma crescente, em relação à presença da função avaliada. Para obter o escore total, é necessário realizar a soma dos valores das questões e multiplicar essa soma pelo fator de correção e, então, somar os valores de cada domínio. Finalizando, o escore 
final da função sexual pode variar entre 2 e 36 , e quanto maior o escore, maior a função, porém valores iguais ou abaixo de 26 pontos são indicadores de disfunção, indicando que a participante apresenta um risco de desenvolver disfunção sexual ${ }^{21}$. Contudo, esse questionário não tem a capacidade de identificar a fase da resposta sexual alterada.

Para avaliar a QV das gestantes, foi utilizado um instrumento que visa avaliar de forma individual e íntima. O Índice de Qualidade de Vida de Ferrans \& Powers. Esse questionário é dividido em quatro dimensões:

1. saúde/funcionamento;

2. psicológico/espiritual;

3. socioeconômico; e

4. família.

Neste estudo foi utilizado o questionário específico para gestantes, composto por 36 questões e dividido em 2 partes: satisfação e importância. Para responder às perguntas, as mulheres atribuíram valores em uma escala de 1 a 6 de satisfação e importância. Para calcular e obter os escores, cada item de satisfação é analisado pelo correspondente de importância, resultando em valores combinados. Dessa forma, os valores mais altos de importância representam alta satisfação e alta importância e os mais baixos, baixa satisfação e alta importância. Por fim, o escore total do questionário varia entre 0 e 30 , com valores altos sendo indicativo de melhor QV.

Os dados coletados da pesquisa foram tabulados em um banco de dados no software Statistical Package for Social Sciencies for Personal Computer (SPSS-PC), versão 20.0. Foi realizada uma análise estatística descritiva, visando uma caracterização da amostra estudada, através das medidas de tendência central (mediana), com seus respectivos valores de dispersão (intervalo interquartis), bem como frequências absolutas e relativas. Foi realizada a verificação da distribuição de normalidade das variáveis pelo teste de Shapiro-Wilk. Para comparar a QV entre as gestantes com e sem disfunção foi realizado o teste de Mann-Whitney, e para comparar a frequência da relação da frequência da atividade sexual mensal do casal antes e durante a gestação, foi realizado o teste de Wilcoxon. Para todas as análises foi adotado para significância dos dados um $\mathrm{p}<0,05$.

\section{Resultados}

No presente estudo, as gestantes avaliadas apresentaram idade mediana de 30 anos (quartil 25\%: 26 anos; quartil 75\%: 33 anos). A maioria das voluntárias se declarou branca $(50,2 \%)$, vivia com o companheiro $(99,0 \%)$, tinha ensino superior $(53,9 \%)$ e possuía renda superior a quatro salários mínimos $(41,3 \%)$. A média da idade gestacional foi de 26 semanas (quartil 25\%: 20 semanas; quartil 75\%: 31 semanas). As demais características socioeconômicas e obstétricas podem ser observadas na Tabela 1 .

Em relação à análise da frequência das relações sexuais antes da gestação, foi constatada uma mediana de 12 vezes por mês, com seu quartil (25-75\%) de 8-16, respectivamente. Entretanto, a frequência da atividade sexual, durante a gestação, teve uma mediana de 4 vezes por mês, com seu quartil (25-75\%) de 4-8 vezes, respectivamente. Essa diminuição da atividade sexual do casal, durante a gestação, foi estatisticamente significativa $(\mathrm{Z}=-10,5 ; \mathrm{p}<0,001)$.

$\mathrm{Na}$ Tabela 2, é possível observar as questões referentes aos domínios sexuais antes e durante a gestação. Foi observada uma diminuição em relação ao desejo e excitação durante a gestação, relatados pelas voluntárias. A maioria conseguia atingir o orgasmo $(74,4 \%)$ e não sentiam dor na relação $(67,5 \%)$ durante a gestação. Das gestantes avaliadas, 65,2\% apresentaram manutenção ou aumento da satisfação sexual durante a gestação.

Neste estudo, ainda, foi verificada uma frequência de $35,7 \%$ das gestantes com presença de disfunção sexual, de acordo com a pontuação do IFSF. Ao comparar os

Tabela 1. Distribuição das variáveis socioeconômicas e obstétricas das gestantes ( $n=207$ )

\begin{tabular}{|c|c|c|}
\hline \multirow{2}{*}{ Variáveis } & \multicolumn{2}{|c|}{ Total } \\
\hline & n & $\%$ \\
\hline \multicolumn{3}{|l|}{ Cor autodeclarada } \\
\hline Branca & 102 & 50,2 \\
\hline Parda & 44 & 21,7 \\
\hline Morena & 47 & 23,2 \\
\hline Negra & 10 & 4,9 \\
\hline \multicolumn{3}{|l|}{ União estável } \\
\hline $\operatorname{Sim}$ & 205 & 99,0 \\
\hline Não & 2 & 1,0 \\
\hline \multicolumn{3}{|l|}{ Escolaridade } \\
\hline Ensino Fundamental & 40 & 19,4 \\
\hline Ensino Médio & 55 & 26,7 \\
\hline Ensino Superior & 111 & 53,9 \\
\hline \multicolumn{3}{|l|}{ Renda } \\
\hline Até 1 salário & 37 & 18,3 \\
\hline 1-2 salários & 34 & 16,9 \\
\hline 3-4 salários & 47 & 23,4 \\
\hline$<4$ salários & 83 & 41,3 \\
\hline \multicolumn{3}{|l|}{ Gravidez planejada } \\
\hline $\operatorname{Sim}$ & 104 & 51,4 \\
\hline Não & 99 & 48,8 \\
\hline \multicolumn{3}{|l|}{ Trimestre gestacional } \\
\hline Segundo & 93 & 44,9 \\
\hline Terceiro & 114 & 55,0 \\
\hline
\end{tabular}


domínios da função sexual, entre o segundo e terceiro trimestres gestacionais, foi observado que os domínios excitação, orgasmo, satisfação e dor, bem como o escore total, sofreram redução com a progressão da gestação. Contudo, apenas a pontuação do domínio dor foi significativamente menor nas gestantes do terceiro trimestre. Isso indica que essas mulheres apresentam mais desconforto durante o ato sexual no último trimestre gestacional.

Foi observado que a presença de disfunção sexual promove uma diminuição estatisticamente significativa da $\mathrm{QV}(\mathrm{Z}=-2,8 ; \mathrm{p}=0,004)$. Ao se comparar a $\mathrm{QV}$ e a presença de disfunção sexual nas gestantes, foram observados resultados estatisticamente significativos para os domínios socioeconômico, psicológico e espiritual. Dentre os domínios da QV avaliados, a mediana do domínio família foi o que o obteve a maior pontuação $(26,4)$, seguido pelo quesito psicológico e espiritual $(25,0)$, socioeconômico $(22,6)$ e, por fim, saúde e funcionamento $(20,8)$ (Tabela 3$)$.

Tabela 2. Caracterização dos domínios sexuais antes e durante a gestação (n=207)

\begin{tabular}{|c|c|c|c|}
\hline Variáveis & $\begin{array}{c}\text { Antes da gestação } \\
\text { n (\%) }\end{array}$ & Variáveis & $\begin{array}{c}\text { Durante a gestação } \\
\text { n (\%) }\end{array}$ \\
\hline Desejo & & Diminuiv & $114(55,3)$ \\
\hline Sim & $190(92,2)$ & Aumentou & $38(18,4)$ \\
\hline Não & $16(7,8)$ & Não alterou & $54(26,2)$ \\
\hline Exciłação & & Diminuiu & $93(44,9)$ \\
\hline Sim & $198(96,6)$ & Aumentou & $29(14,0)$ \\
\hline Não & $7(3,4)$ & Não alterou & $85(41,1)$ \\
\hline \multicolumn{4}{|l|}{ Orgasmo } \\
\hline Sim & $193(94,1)$ & Sim & $151(74,4)$ \\
\hline Não & $12(5,9)$ & Não & $52(25,6)$ \\
\hline \multicolumn{4}{|l|}{ Dor } \\
\hline Sim & $40(19,5)$ & Sim & $66(32,5)$ \\
\hline Não & $165(80,5)$ & Não & $137(67,5)$ \\
\hline Satisfação & & Diminuiv & $71(34,8)$ \\
\hline Sim & $202(98,5)$ & Aumentou & $17(8,3)$ \\
\hline Não & $3(1,5)$ & Não alterou & $116(56,9)$ \\
\hline
\end{tabular}

Tabela 3. Comparação dos domínios da qualidade de vida entre as gestantes com e sem disfunção sexual

\begin{tabular}{|c|c|c|c|c|}
\hline $\begin{array}{l}\text { Domínios da } \\
\text { qualidade de vida }\end{array}$ & $\begin{array}{c}\text { Com } \\
\text { disfunção } \\
\text { sexual }\end{array}$ & $\begin{array}{c}\text { Sem } \\
\text { disfunção } \\
\text { sexual }\end{array}$ & Z & Valor $\mathrm{p}$ \\
\hline Saúde e funcionamento & 20,1 & 21,0 & $-1,1$ & 0,28 \\
\hline Socioeconômico & 21,7 & 23,8 & $-3,5$ & $0,001^{*}$ \\
\hline Psicológico e espiritual & 24,2 & 25,7 & $-2,5$ & $0,01^{*}$ \\
\hline Familia & 26,4 & 26,4 & $-1,0$ & 0,30 \\
\hline Total & 22,2 & 24,2 & $-2,9$ & $0,004^{*}$ \\
\hline
\end{tabular}

${ }^{*} p<0,05$

\section{Discussão}

Os resultados encontrados neste estudo confirmam que a presença de disfunção sexual pode interferir negativamente na QV de gestantes. Estudos prévios verificaram que a função sexual está associada negativamente com a QV de mulheres grávidas ${ }^{22,23}$.

A avaliação da QV é uma tarefa complexa, em função da sua multidimensionalidade e facetas positivas e negativas entre os quesitos avaliados ${ }^{24}$. Neste estudo, a QV entre as gestantes avaliadas foi considerada relativamente boa. O domínio família foi o que obteve a maior pontuação, seguido pelo quesito psicológico e espiritual, socioeconômico e, por fim, saúde e funcionamento. A explicação para esse achado pode ser devido ao fato de $99,0 \%$ das participantes do estudo estarem em uma união estável e com o apoio familiar na gravidez.

$\mathrm{O}$ apoio social é um construto multidimensional, que envolve conforto, carinho, assistência e/ou informações recebidos de contatos sociais. Relaciona-se com a percepção do indivíduo acerca do seu mundo social, com as estratégias para estabelecer vínculos e os recursos que lhe são oferecidos ${ }^{25}$. O papel do apoio social nas diversas fases da vida é fundamental para o amortecimento de eventos estressantes que ocorrem no cotidiano, principalmente em momentos em que se observam diversas modificações psicossociais e fisiológicas, como é o caso da gestação.

Neste estudo, observou-se uma diminuição significativa da QV das gestantes com disfunção sexual. Estudos têm demonstrado que, durante o período gestacional, a QV está sujeita a sofrer alterações ${ }^{1,20,26}$. Tal ocorrência pode estar relacionada a fatores sociodemográficos como: idade, nível educacional, planejamento familiar, carga horária de trabalho e acompanhamento pré-natal. Tais fatores, além de mitos e tabus sobre o sexo na gravidez, podem afetar o relacionamento do casal. Dentre as variáveis obstétricas, a idade gestacional parece ser o fator que causa maior influência sobre a QV, interferindo nos comportamentos da grávida, relacionada à sua funcionalidade, socialização e atividades rotineiras ${ }^{27}$. A função sexual sofre mudança. Muitas vezes, ocorre o aumento dos sintomas de disfunção sexual, como diminuição de desejo e dispareunia nesse período, o que pode interferir diretamente na satisfação, felicidade e QV em grávidas.

Nesta pesquisa, a mediana da idade encontrada foi relativamente alta, sugerindo uma população de gestantes considerada mais madura. Dados da literatura têm demonstrado que a disfunção sexual é frequentemente encontrada em grávidas que possuem idade avançada, quando comparada com as gestantes mais jovens ${ }^{19}$. Estudo prévio ${ }^{28}$, entretanto, avaliando a 
QV, verificou que a idade mais avançada das gestantes pode estar associada com uma melhor saúde mental. Tal fato poderia interferir de forma positiva na QV, uma vez que tais mulheres, devido às diversas vivências experimentadas, podem desenvolver métodos de enfrentamento e usá-los para lidar da melhor forma com os problemas emocionais.

O segundo trimestre gestacional é um período emocionalmente estável, comparado aos demais, pois a gravidez encontra-se estabelecida. Isso aumenta a segurança dos pais a respeito de possíveis perdas fetais, associadas às alterações vasculares na região genital e à cessação dos incômodos iniciais. Essas alterações permitem um aumento da resposta sexual dessas mulheres, apresentando melhoria na qualidade orgástica e no comportamento sexual ${ }^{4,19,29}$.

O desejo sexual é o primeiro ponto de encontro para vivência da sexualidade na gestação. Nesse período, a mulher vive um conflito, pois se encontra em um momento de transição, mudança e de identificação com o próprio corpo. Neste estudo, 91,7\% das mulheres sentiam desejo sexual antes da gestação. Contudo, durante a gestação 56,6\% dessas mulheres tiveram seu desejo sexual diminuído. Esse fato pode ser explicado pela ambivalência de sentimentos, apresentado nas grávidas. As mulheres, durante o período gestacional, podem se tornar vulneráveis às influências socioculturais, quando devem aceitar em seu corpo a geração de um ser. Ao mesmo tempo, esse corpo grávido deseja manter relações sexuais ${ }^{4,23,29-31}$.

De acordo com a literatura, o desejo sexual aumenta do primeiro para o segundo trimestre e, em seguida, diminui novamente, à medida que a gravidez avança $a^{4,23,24,29,30,32}$. Confirmando esses achados, os resultados do presente estudo demonstraram que existe uma diminuição nos valores do escore do ISFS das gestantes do último trimestre quando comparado ao das gestantes do segundo trimestre.

Esses resultados podem se dever ao fato de que, nas últimas semanas de gestação, o desconforto, a falta de ar, a fadiga, o aumento de tamanho do bebê, o seu encaixe na pelve e a pressão pélvica podem tornar a relação sexual menos confortável e, portanto, o desejo é diminuído ${ }^{30,32}$.

As mudanças ocorridas no último trimestre da gravidez podem ser a razão para a diminuição da libido e da atividade sexual durante esse período gestacional ${ }^{4,23,29,30}$. O peso fetal e o volume abdominal aumentados causam alteração do centro de gravidade, alterando o equilíbrio e acarretando modificações posturais compensatórias. Isso exige do organismo feminino o uso de musculaturas acessórias, pouco recrutadas no período pré-gestacional, para manter a postura e o equilíbrio. Esse fato pode gerar o aparecimento de desconfortos e dores, principalmente na região lombar. Além disso, a fadiga, a ansiedade e o medo natural, devido à proximidade do trabalho do parto, tendem a tornar a relação sexual sem atrativos para as mulheres grávidas. Outro fator que contribui para diminuir a função sexual feminina é a perda do interesse sexual do parceiro, por causa de preocupações com a mulher e o bebê, bem como o efeito não erótico da aparência da mulher no final da gravide $z^{19}$.

Os resultados obtidos neste estudo demonstraram uma diminuição estatisticamente significativa da frequência da relação sexual, antes e durante a gravidez. Resultados semelhantes têm sido demonstrado por outros estudos, que sugerem que a frequência da atividade sexual tende a declinar durante a gestação, em relação ao período pré-gestacional e ao próprio período gestacional ${ }^{3,4,15-17,23,29,30,32,33}$. Estudos enfatizam que a diminuição da frequência da atividade sexual pode ser decorrente do temor de prejudicar o feto, mal-estar e desconforto corporal e perda do interesse em atividade sexual. Além disso, o receio em antecipar o parto, incômodos posturais e dor, aumento do volume abdominal e distorção na imagem corporal podem ser também fatores responsáveis pelo declínio da atividade sexual na progressão da gestação ${ }^{16,17,33}$.

As gestantes avaliadas neste estudo revelaram estar sexualmente satisfeitas antes da gravidez e que sua satisfação sexual não se alterou ou sofreu um aumento durante o período gestacional. Estudo prévio ${ }^{32}$ afirmou que a satisfação plena das mulheres se correlaciona positivamente com o sentimento de felicidade em estar grávida, principalmente se a gestação for desejada e planejada.

A redução do desejo, da atividade sexual e do coito vaginal ocorre, em muitas mulheres, com o avançar da gravidez, pois tanto a mulher quanto seu parceiro têm preocupações sobre complicações na gestação como resultado de relações sexuais ${ }^{3}$. A maioria das mulheres gostaria de discutir esses problemas com seu médico, mas nem sempre se sentem confortáveis ${ }^{32}$. A sexualidade e a satisfação sexual não estão restritas ao ato sexual em si. As atividades sexuais não provocam danos, efeitos adversos ou indução ao trabalho de parto à gravidez normal, podendo beneficiá-la, aliviando a ansiedade e a tensão, através da satisfação sexual ${ }^{29}$.

Como limitações deste estudo, há a ausência, na amostra, de mulheres no primeiro trimestre gestacional, o que pode ser justificado pelo fato de que muitas dessas mulheres descobrem a gravidez somente a partir do início do segundo trimestre. Isso demonstra uma falta de conscientização por parte das próprias grávidas da importância do início precoce do pré-natal e comparecimento às consultas. Outra limitação encontrada em nosso estudo foi o fato do parceiro não ter sido incluído na pesquisa, 
uma vez que a diminuição na função sexual pode estar também relacionada ao interesse do cônjuge.

Este estudo mostrou que a QV das gestantes pode diminuir devido a alterações na função sexual acarretadas pela progressão da gestação. Trata-se de um aspecto relevante, pouco avaliado na prática clínica pré-natal. Deve-se, portanto, enfatizar a importância da avaliação e orientação à grávida e ao seu parceiro da sexualidade na gravidez, promovendo assim uma melhora na QV da mulher durante o período gestacional.

\section{Referências}

1. Tendais I, Figueiredo B, Mota J, Conde A. Physical activity, healthrelated quality of life and depression during pregnancy. Cad Saúde Pública. $2011 ; 27(2): 219-28$.

2. Couto ER, Couto E, Vian B, Gregório Z, Nomura ML, Zaccaria $R$, et al. Quality of life, depression and anxiety among pregnant women with previous adverse pregnancy outcomes. Sao Paulo Med J. 2009; 127(4): 185-9.

3. Corbacioglu Esmer A, Akca A, Akbayir O, Goksedef BP, Bakir VL. Female sexual function and associated factors during pregnancy. J Obstet Gynaecol Res. 2013;39(6):1 165-72.

4. Ahmed MR, Madny EH, Sayed Ahmed WA. Prevalence of female sexual dysfunction during pregnancy among Egyptian women. J Obstet Gynaecol Res. 2014;40(4): 1023-9.

5. Lima AC, Dotto LMG, Mamede MV. Prevalência de disfunção sexual em primigestas, no Município de Rio Branco, Acre, Brasil. Cad Saúde Pública. 2013;29(8):1544-54.

6. Mimoun S, Wylie K. Female sexual dysfunctions: definitions and classification. Maturitas. 2009;63(2):1 16-8.

7. Jah S, Thakar R. Female sexual dysfunction. Eur J Obstet Reprod Biol. 2010;153(2):117-23.

8. Ribeiro MC, Nakamura MU, Torloni MR, Scanavino MT, Scomparini FB, Mattar R. Female sexual function of overweight women with gestational diabetes mellitus: a cross-sectional study. PLoS One. 2014;9(4):e95094.

9. Murtagh J. Female sexual function, dysfunction, and pregnancy: implications for practice. J Midwifery Womens Health. 2010;55(5):438-46.

10. Tunuguntla HS, Gousse AE. Female sexual dysfunction following vaginal surgery: a review. J Urol. 2006;175(2):439-46.

11. Raina R, Pahlajani G, Khan S, Gupta S, Agarwal A, Zippe CD. Female sexual dysfunction: classification, pathophysiology, and management. Fertil Steril. 2007;88(5):1273-84.

12. Lara LA, Rosa e Silva AC, Romão AP, Junqueira FR. [The assessment and management of female sexual dysfunction]. Rev Bras Ginecol Obstet. 2008;30(6):312-21. Portuguese.

13. Rosen RC. Assessment of female sexual dysfunction: review of validated methods. Fertil Steril. 2002;77(Suppl 4):S89-93.

14. Knoepp LR, Shippey SH, Chen CC, Cundiff GW, Derogatis LR, Handa VL. Sexual complaints, pelvic floor symptoms, and sexual distress in women over forty. J Sex Med. 2010;7(1 1):3675-82.

15. Eryilmaz G, Ege E, Zincir H. Factors affecting sexual life during pregnancy in eastern Turkey. Gynecol Obstet Invest. 2004;57(2): 103-8.

16. Aslan G, Aslan D, Kizilyar A, Ispahi C, Esen A. A prospective analysis of sexual functions during pregnancy. Int J Impot Res. 2005; 17(2): 154-7.

17. Babazadeh R, Najmabadi KM, Masomi Z. Changes in sexual desire and activity during pregnancy among women in Shahroud, Iran. Int J Gynaecol Obstet. 2013;120(1):82-4.
18. Prado DS, Lima RV, Lima LM. [Impact of pregnancy on female sexual function]. Rev Bras Ginecol Obstet. 2013;35(5):2059. Portuguese.

19. Leite AP, Campos AA, Dias AR, Amed AM, Souza E, Camano L. Prevalence of sexual dysfunction during pregnancy. Rev Assoc Med Bras. 2009;55(5):563-8.

20. Lau Y, Yin L. Maternal, obstetric variables, perceived stress and health-related quality of life among pregnant women in Macao, China. Midwifery. $2011 ; 27(5): 668-73$.

21. Leite APL, Moura EA, Campos AAS, Mattar R, Souza E, Camano L. [Validation of the Female Sexual Function Index in Brazilian pregnant women]. Rev Bras Ginecol Obstet. 2007;29(8):396401. Portuguese.

22. Ferreira $D Q$, Nakamura $M U$, Souza E, Mariani Neto $C$, Ribeiro $M C$, Santana TGM, et al. [Sexual function and quality of life of low-risk pregnant women]. Rev Bras Ginecol Obstet. 2012;34(9):409-13. Portuguese.

23. Gałązka I, Drosdzol-Cop A, Naworska B, Czajkowska M, SkrzypulecPlinta $V$. Changes in the sexual function during pregnancy. J Sex Med. 2015;12(2):445-54.

24. Gökyildiz S, Beji NK. The effects of pregnancy on sexual life. J Sex Marital Ther. 2005;31(3):201-15.

25. Thiengo DL, Santos JFC, Fonseca DL, Abelha L, Lovisi GM. Depressão durante a gestação: um estudo sobre a associação entre fatores de risco e de apoio social entre gestantes. Cad Saúde Coletiva. 2012;20(4):416-26.

26. Eufrásio LS, Sousa VPS, Viana ESR. Análise da relação entre idade gestacional e qualidade de vida em gestantes atendidas em unidades básicas de saúde da cidade do Natal. Fisioter Bras. 2012;13(Suppl 6):185-9.

27. Tašdemir S, Balci E, Günay $O$. Comparison of life quality of pregnant adolescents with that of pregnant adults in Turkey. Ups J Med Sci. 2010;1 15(4):275-81

28. Li J, Mao J, Du Y, Morris JL, Gong G, Xiong X. Health-related quality of life among pregnant women with and without depression in Hubei, China. Matern Child Health J. 2012;16(7):1355-63.

29. Jamali S, Mosalanejad L. Sexual dysfunction in Iranian pregnant women. Iran J Reprod Med. 2013;1 1 (6):479-86.

30. Hanafy S, Srour NE, Mostafa T. Female sexual dysfunction across the three pregnancy trimesters: an Egyptian study. Sex Health. 2014; $11(3): 240-3$

31. Prado DS, Mota VP, Lima TI. [Prevalence of sexual dysfunction in two women groups of different socioeconomic status]. Rev Bras Ginecol Obstet. 2010;32(3):139-43. Portuguese.

32. Khamis MA, Mustafa MF, Mohamed SN, Toson MM. Influence of gestational period on sexual behavior. J Egypt Public Health Assoc. 2007;82(1-2):65-90.

33. Yeniel AO, Petri E. Pregnancy, childbirth and sexual function: perceptions and facts. Int Urogynecol J. 2014;25(1):5-14. 